\title{
Delivery of a Novel Connexin-43 Mimetic Peptide Enhances Wound Healing
}

\author{
K. Moore ${ }^{1}$, Z. J. Bryant ${ }^{1}$, A. Vandergriff ${ }^{1}$, G. Ghatnekar ${ }^{2}$, R. Gourdie ${ }^{3}$, J. Potts ${ }^{1}$
}

${ }^{1}$ University of South Carolina School of Medicine, Department of Cell Biology and Anatomy, Columbia, South Carolina 29209, USA

${ }^{2}$ FirstString Research Inc., Charleston, South Carolina 29403, USA

${ }^{3}$ Virginia Polytechnic and State University Carilion, VTC Research Institute, Roanoke, Virginia 24016, USA

The ability to safely and quickly close wounds and lacerations is an area of need in regenerative medicine, with implications toward healing a wide range of tissues and wounds. Using a number in vivo injury models, our lab has investigated a newly developed peptide capable of promotion of wound healing and epithelial regeneration. The alpha-carboxy terminus $1(\alpha \mathrm{CT} 1)$ peptide is a 25 amino acid peptide from the C-terminus of connexin 43 (Cx43), modified to promote cellular uptake. Previous studies applying $\alpha \mathrm{CT} 1$ to excisional wounds in porcine models produced tissues having an overall reduced level of scar tissue and decreased healing time. Early studies in our laboratory applying $\alpha \mathrm{CT} 1$ to excisional wounds in rats resulted in tissue having an overall reduced level of scar formation and decreased healing time, compared to controls. Both $\alpha \mathrm{CT} 1$ alone and in combination with bone marrow stromal cells (BMSCs) were used in the treatment of excisional wounds. The interplay between the two agents was investigated and demonstrated that stem cells in addition to $\alpha \mathrm{CT} 1$ proved to be very effective in closing wounds quicker and with less scar tissue present (Fig.1).

Recently we have investigated methods of enhancing the delivery method of the $\alpha \mathrm{CT} 1$ to various wounds. Microencapsulation of the $\alpha \mathrm{CT} 1$ peptide using an alginate polymer provides a novel timerelease formula for the peptide. In our rat corneal wound model, we delivered $\alpha \mathrm{CT} 1$ both directly, in a concentrated pluronic solution, and in a sustained system, using polymeric alginate-poly-1-ornithine (APLO) microcapsules. Cell toxicity analysis showed minimal cell-loss with microcapsule treatment. Measurement of wound healing using histology and fluorescence microscopy indicated significant reduction in healing time of $\alpha \mathrm{CT} 1$ microcapsule treated rat corneas compared with controls $(88 \%$ vs. $38 \%$ ). RT-PCR analysis of treated corneas showed an initial up regulation followed by down regulation of the gene keratin-19 (Krt19). Zonula occluden 1 (ZO-1) showed an opposite down regulation followed by an up regulation whereas $\mathrm{Cx} 43$ showed a biphasic response. Inflammatory indexes demonstrated a reduction in the inflammation of corneas treated with $\alpha \mathrm{CT} 1$ microcapsules when compared with pluronic gel vehicle (Fig. 2). Finally, we employed a scratch wound assay to investigate the mechanism of action of $\alpha \mathrm{CT} 1$ peptide. We examined scratched BMSCs for a number of genes involved in wound healing. Krt19 was again upregulated in contrast to Cx43, ZO-1, Snail 2, and JamA which all showed a down regulation of transcript expression. In total these results begin to lay the foundation for future therapeutic application of $\alpha \mathrm{CT} 1$ as a beneficial wound healing treatment. 


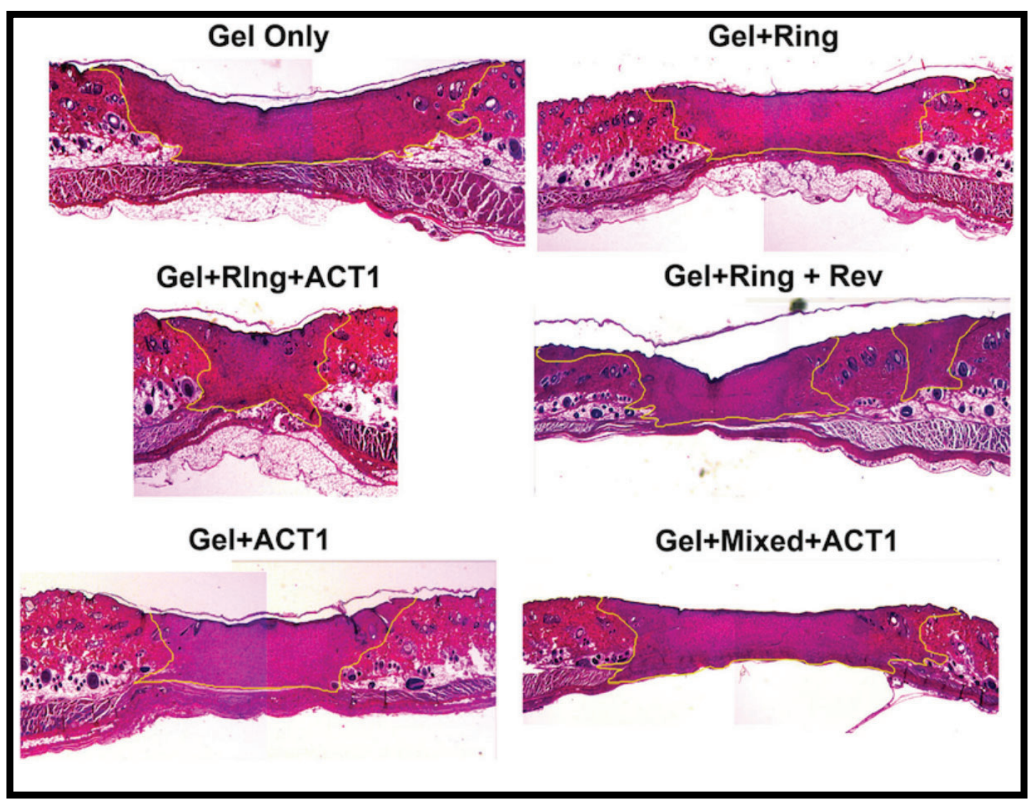

Figure 1. Excisional skin wounds 30 days post surgery stained for the presence of scar tissue. Skin wounds were treated with a regime of $\alpha \mathrm{CT} 1$ alone and in combination with BMSCs. The treatment of $\alpha \mathrm{CT} 1$ in combination with Stem cells grown to form a toroidal (ring) showed significant improvement compared with the other treatments.

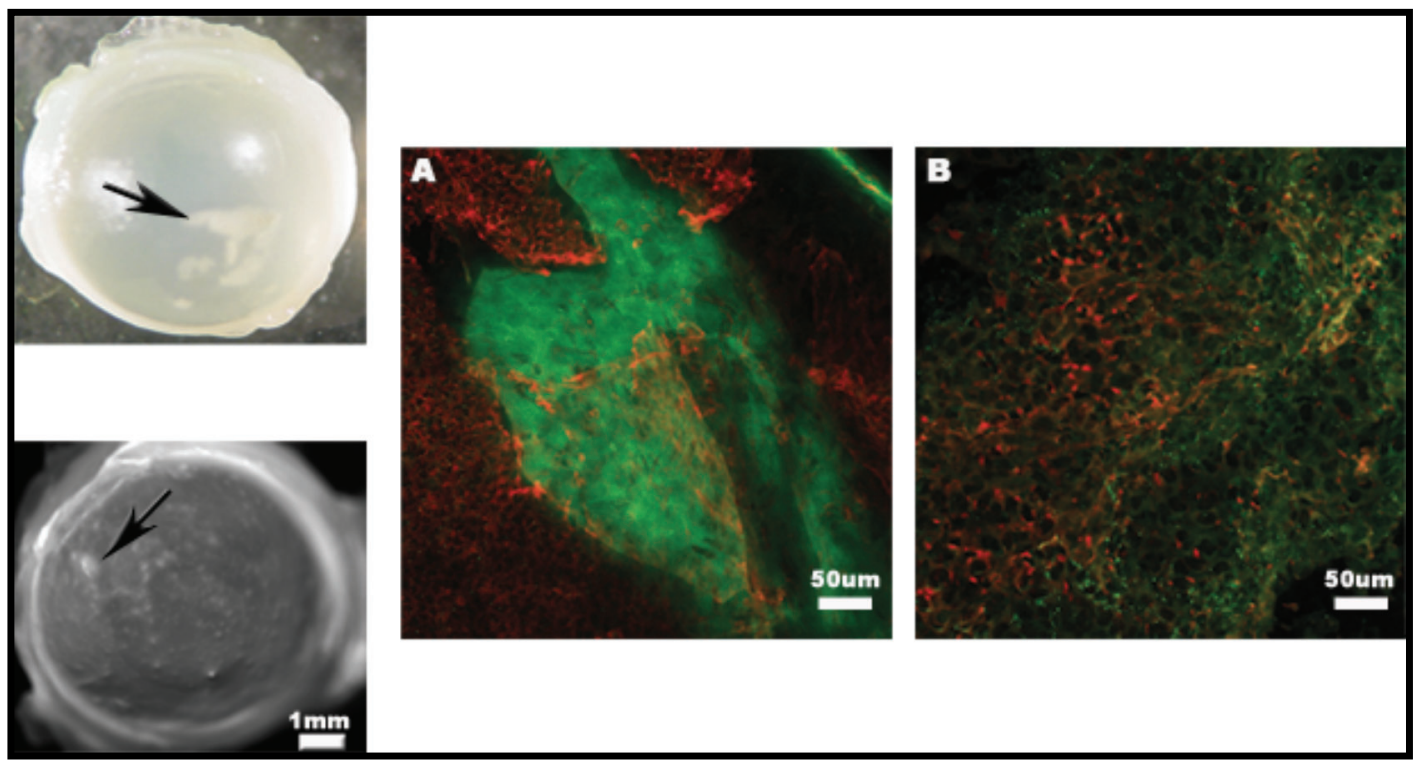

Figure 2. The inflammatory response of the rat cornea to the ACT1 A-PLO microcapsule treatment. Images are shown in both the upper left and lower left of the figure showing two representative portions of the affected tissue of the cornea at 30 days. (A) Confocal image at a 20x magnification of the affected tissue in a 3 day microcapsule treated cornea. Green-TNF- $\alpha$, Red-smooth muscle actin. (B) 20x magnification of the affected tissue in a 3 day microcapsule treated cornea. Green-ITAC, Red-smooth

[1] K Moore et al,. Microscopy and Microanalysis. (In Press) 2013

[2] The authors acknowledge funding from NIH 5K02HL086901and First String Research, Inc. Dr. R Goodwin is thanked for his many useful discussions to this work 